\title{
EDITORIAL
}

\section{Personalised medicine in exacerbations of COPD: the beginnings}

\author{
Sanjay Sethi
}

$\mathbf{E}$ xacerbations of chronic obstructive pulmonary disease (COPD) are inflammatory events induced by infection [1]. Therefore, the use of antimicrobial and antiinflammatory medications in exacerbations appears logical. However, the evidence that antibiotics and corticosteroids are beneficial in the treatment of exacerbations is relatively sparse, and only a meta-analytic approach has given us sufficient confidence in the use of these treatments [2, 3]. Placebocontrolled and observational studies in hospitalised patients with COPD exacerbations have shown the benefit of antibiotics and steroids [4-6]. This has led to current recommendations for treating all hospitalised exacerbations with both antibiotics and steroids. However, as we appreciate the heterogeneity of infection and inflammation in exacerbations, using the same treatment in all exacerbations does not appear appropriate. Bacterial infection causes approximately $50 \%$ of exacerbations; therefore, antibiotic use is probably unnecessary in the other $50 \%$ [1]. Eosinophilic inflammation is seen in a third of exacerbations, so corticosteroids may be of limited benefit in a substantial proportion of exacerbations [7].

In a hospitalised patient with a COPD exacerbation, the cost of antibiotics accounts for a small fraction of the total cost of care. However, everyone is well aware of the dangers of inappropriate and excessive antibiotic use with regards to selection of antibiotic resistant pathogens and adverse effects. Therefore, discriminating exacerbations that are bacterial in origin from those that are not would help us target antibiotic therapy. Sputum cultures lack the sensitivity, specificity and speed to be such a discriminatory tool. Biomarkers such as procalcitonin and C-reactive protein could be useful, but are costly (for procalcitonin), lack proper microbiological and clinical validation, and, as illustrated by SOLER et al. [8], perform quite differently in various studies and populations [9]. Sputum purulence can be regarded as a biomarker of a bacterial exacerbation. It is attractive in many respects as it is cheap, has been validated microbiologically, and is available at the point of care $[10,11]$. What it lacks is proper clinical validation as a guide for the need of antibiotic treatment.

Division of Pulmonary, Critical Care and Sleep Medicine, University at Buffalo, State University of New York, Buffalo, NY, USA.

CORRESPONDENCE: S. Sethi, Division of Pulmonary, Critical Care and Sleep Medicine, University at Buffalo, State University of New York, VA Medical research - 151, 3495 Bailey Ave, Buffalo, NY 14051, USA. E-mail: ssethi@buffalo.edu
Although assessing sputum purulence should be straightforward, that is not always the case. Sputum purulence is reliable in patients with baseline mucoid (non-purulent) sputum. However, a third of patients with COPD may have baseline purulent sputum [12]. In those patients, an increase in purulence can sometimes be discerned, but not always. Patients often do not pay attention to the colour of sputum or describe dynamic changes in purulence: "It was yellow last night, but looked clear today morning". And then there are those with very severe airflow obstruction who feel chest congestion but cannot bring up the sputum: "I hear it rattling in my chest but I cannot get it up, so I do not know what colour it is".

In their unique and well-conducted trial, SOLER et al. [8] have attempted to address the clinical utility of sputum purulence as a determinant of antibiotic treatment in hospitalised exacerbations of COPD. The design of this study is unique in that antibiotics were withheld in patients without purulent sputum and the comparator group was composed of patients with purulent exacerbations treated with antibiotics. Of note, only 73 out of 184 eligible patients met the inclusion and exclusion criteria for the study, with prior recent antibiotic and corticosteroid use and need for intensive care being the most common (and appropriate) reasons for exclusion. Most patients received systemic corticosteroids. The primary outcome of treatment failure was measured at day 3 , and was comparable in the two groups.

Does this result mean than we should withhold antibiotics in hospitalised patients with COPD exacerbations based on the absence of purulent sputum? Although this study would certainly suggest that is appropriate, there are issues of study design that should give us pause. In clinical studies, when groups are compared, randomisation is essential to provide baseline equivalence between the two groups, so that we are comparing "apples to apples". Furthermore, placebo controls and blinding are also essential. In this study, whether patients with purulent sputum (bacterial) exacerbations were the same as those without purulent sputum (non-bacterial) is debatable. There is now ample evidence in the literature that bacterial exacerbations are associated with greater levels of airway and systemic inflammation and greater clinical severity than nonbacterial exacerbations [13, 14]. In their landmark study, ANTHONISEN et al. [15] showed that type 1 exacerbations (with increased dyspnoea, sputum volume and sputum purulence) had a much lower rate of spontaneous resolution in the placebo arm compared to type 2 (two of the three cardinal 
symptoms) or type 3 (one of the three cardinal symptoms) exacerbations, with rates of 43,60 and $69.7 \%$, respectively. Because of the design of this study, there is a striking imbalance between the two arms in the Anthonisen type of exacerbations, with $82 \%$ versus $0 \%$ being type 1 and 0 versus $47 \%$ being type 3 in the purulent and non-purulent groups, respectively. Furthermore, at day $30,74 \%$ of the non-purulent exacerbations had achieved complete clinical resolution, compared to only $46 \%$ of the purulent group. At day 180, $36 \%$ of the non-purulent group had experienced a recurrent exacerbation, while this happened in as many as $60 \%$ of purulent group. Systemic inflammation, as measured by serum C-reactive protein, was significantly greater in the purulent group at admission and on day 3 . Therefore, comparison of these two groups of patients is of "apples to oranges".

If the comparison of the two groups is confounded by baseline differences, what does this study teach us? More important than the comparison between the two groups of patients is the observation of good clinical outcomes in the non-purulent group. This study essentially extends the observation of the Anthonisen study to hospitalised patients, in that patients with type 3 and selected type 2 exacerbations may not benefit from antibiotics $[1,15]$. This study provides the basis of performing a randomised placebo controlled trial of antibiotics in hospitalised patients with non-purulent sputum, which appears to be underway, which will give us complete confidence in withholding antibiotics in such patients.

Can we better target systemic steroid use in exacerbations? Although a short course of systemic steroids is often regarded as benign, there is emerging evidence that this may not be the case in the setting of an exacerbation of COPD. The best described adverse effect is hyperglycaemia [6]. High-dose systemic steroids for exacerbations have been associated with invasive aspergillosis, a disorder with high morbidity and mortality [16]. Two recent trials have raised additional concerns. In the MAESTRAL study, comparing moxifloxacin to amoxicillin/clavulanate in out-patient type 1 exacerbations of COPD, about $35 \%$ of patients received systemic corticosteroids [17]. These patients experienced worse outcomes than those who did not receive systemic corticosteroids, although they were not discernibly sicker than patients who did not receive systemic steroids. In another recent study, patients with exacerbations of COPD who did not have eosinophilic inflammation at exacerbations and received systemic corticosteroids had worse outcomes, while those with eosinophilic inflammation were shown to have benefit with steroids [18].

Given the medical and economic importance of exacerbations of COPD, our evidence base for appropriate management of this common problem has been lamentable. However, this study and other work gives us good reason to hope that we will be practicing personalised medicine in exacerbations of COPD in the near future, where, based on clinical assessment or easily obtained biomarkers, we will be selecting who will receive antibiotics and systemic steroids.

\section{STATEMENT OF INTEREST}

A statement of interest for $\mathrm{S}$. Sethi can be found at www.erj.ersjournals. $\mathrm{com} / \mathrm{misc} / \mathrm{site} /$ statements.xhtml

\section{REFERENCES}

1 Sethi S, Murphy TF. Infection in the pathogenesis and course of chronic obstructive pulmonary disease. N Engl J Med 2008; 359: 2355-2365.

2 Ram FS, Rodriguez-Roisin R, Granados-Navarrete A, et al. Antibiotics for exacerbations of chronic obstructive pulmonary disease. Cochrane Database Syst Rev 2006; 2: CD004403.

3 Wood-Baker RR, Gibson PG, Hannay M, et al. Systemic corticosteroids for acute exacerbations of chronic obstructive pulmonary disease. Cochrane Database Syst Rev 2005; 1: CD001288.

4 Nouira S, Marghli S, Belghith M, et al. Once daily oral ofloxacin in chronic obstructive pulmonary disease exacerbation requiring mechanical ventilation: a randomised placebo-controlled trial Lancet 2001; 358: 2020-2025.

5 Rothberg MB, Pekow PS, Lahti M, et al. Antibiotic therapy and treatment failure in patients hospitalized for acute exacerbations of chronic obstructive pulmonary disease. JAMA 2010; 303: 2035-2042.

6 Niewoehner DE, Erbland ML, Deupree RH, et al. Effect of systemic glucocorticoids on exacerbations of chronic obstructive pulmonary disease. Department of Veterans Affairs Cooperative Study Group. N Engl J Med 1999; 340: 1941-1947.

7 Bafadhel M, McKenna S, Terry S, et al. Acute exacerbations of chronic obstructive pulmonary disease: identification of biologic clusters and their biomarkers. Am J Respir Crit Care Med 2011; 184: 662-671.

8 Soler N, Esperatti M, Ewig S, et al. Sputum purulence-guided antibiotic use in hospitalised patients with exacerbations of COPD. Eur Respir J 2012; 40: 1344-1353.

9 Stolz D, Christ-Crain M, Bingisser R, et al. Antibiotic treatment of exacerbations of COPD: a randomized, controlled trial comparing procalcitonin-guidance with standard therapy. Chest 2007; 131: 9-19.

10 Stockley RA, O'Brien C, Pye A, et al. Relationship of sputum color to nature and outpatient managment of acute exacerbations of COPD. Chest 2000; 117: 1638-1645.

11 Soler N, Agusti C, Angrill J, et al. Bronchoscopic validation of the significance of sputum purulence in severe exacerbations of chronic obstructive pulmonary disease. Thorax 2007; 62: 29-35.

12 Sethi S, Jones PW, Theron MS, et al. Pulsed moxifloxacin for the prevention of exacerbations of chronic obstructive pulmonary disease: a randomized controlled trial. Respir Res 2010; 11: 10

13 Sethi S, Wrona C, Eschberger K, et al. Inflammatory profile of new bacterial strain exacerbations of chronic obstructive pulmonary disease. Am J Respir Crit Care Med 2008; 177: 491-497.

14 Wilkinson TM, Hurst JR, Perera WR, et al. Effect of interactions between lower airway bacterial and rhinoviral infection in exacerbations of COPD. Chest 2006; 129: 317-324.

15 Anthonisen NR, Manfreda J, Warren CPW, et al. Antibiotic therapy in exacerbations of chronic obstructive pulmonary disease. Ann Intern Med 1987; 106: 196-204.

16 Bulpa P, Dive A, Sibille Y. Invasive pulmonary aspergillosis in patients with chronic obstructive pulmonary disease. Eur Respir J 2007; 30: 782-800.

17 Wilson R, Anzueto A, Miravitlles M, et al. Moxifloxacin versus amoxicillin/clavulanic acid in outpatient acute exacerbations of COPD: MAESTRAL results. Eur Respir J 2012; 40: 17-27.

18 Bafadhel M, McKenna S, Terry S, et al. Blood eosinophils to direct corticosteroid treatment of exacerbations of chronic obstructive pulmonary disease: a randomized placebo-controlled trial. Am J Respir Crit Care Med 2012; 186: 48-55. 